DOI 10.4171/JEMS/340

Michael Hochman

\title{
Geometric rigidity of $\times m$ invariant measures
}

Received September 2, 2010 and in revised form May 5, 2011

\begin{abstract}
Let $\mu$ be a probability measure on $[0,1]$ which is invariant and ergodic for $T_{a}(x)=$ ax $\bmod 1$, and $0<\operatorname{dim} \mu<1$. Let $f$ be a local diffeomorphism on some open set. We show that if $E \subseteq \mathbb{R}$ and $\left.\left.(f \mu)\right|_{E} \sim \mu\right|_{E}$, then $f^{\prime}(x) \in\left\{ \pm a^{r}: r \in \mathbb{Q}\right\}$ at $\mu$-a.e. point $x \in f^{-1} E$. In particular, if $g$ is a piecewise analytic map preserving $\mu$ then there is an open $g$-invariant set $U$ containing supp $\mu$ such that $\left.g\right|_{U}$ is piecewise linear with slopes which are rational powers of $a$.

In a similar vein, for $\mu$ as above, if $b$ is another integer and $a, b$ are not powers of a common integer, and if $v$ is a $T_{b}$-invariant measure, then $f \mu \perp v$ for all local diffeomorphisms $f$ of class $C^{2}$. This generalizes the Rudolph-Johnson Theorem and shows that measure rigidity of $T_{a}, T_{b}$ is a property not of the structure of the abelian action, but rather of their smooth conjugacy classes: if $U, V$ are maps of $\mathbb{R} / \mathbb{Z}$ which are $C^{2}$-conjugate to $T_{a}, T_{b}$ then they have no common measure of positive dimension that is ergodic for both.
\end{abstract}

Keywords. Measure rigidity, invariant measure, interval map, fractal geometry, geometric measure theory, scenery flow

\section{Introduction}

\subsection{Background}

The motivating problem of this paper is to understand, for "structured" Borel probability measures whose support is a Cantor set on $\mathbb{R}$, which transformations can map one measure to another, in whole or in part, and how the structure of the measures determines this. More precisely, writing $f \mu$ for the measure $f \mu(A)=\mu\left(f^{-1} A\right)$, we ask what one can say about maps $f$ for which $f \mu$ is non-singular with respect $\mu$, or with respect to some other measure $v$. The expectation is that highly structured measures should be preserved by a small number of maps whose structure reflects that of the measure.

There are a few elementary things one can say. Since any two non-atomic probability measures on $\mathbb{R}$ can be mapped to each other by a continuous function, one must impose some regularity assumption for the question to make sense. In this paper we consider diffeomorphisms of the line, and denote the set of such maps by $\operatorname{diff}(\mathbb{R})$. Write $\operatorname{diff}^{k}(\mathbb{R})$ for the set of diffeomorphisms of class $C^{k}$. Also, there are some trivial cases of measures $\mu$

M. Hochman: Fine Hall, Washington Rd, Princeton, NJ 08544, USA;

e-mail: mhochman@math.huji.ac.il

Mathematics Subject Classification (2010): 37A10, 37E10, 37A45, 37C45, 28A80 
such that $\mu, f \mu$ are non-singular for many $f \in \operatorname{diff}(\mathbb{R})$, namely if $\mu$ has a Lebesgue component or atoms. One must therefore consider measures without such components. Recall that the local dimension of a measure $\mu$ at $x$ is the limit

$$
D(\mu, x)=\lim _{r \rightarrow 0} \frac{\log \mu\left(B_{r}(x)\right)}{\log r}
$$

where $B_{r}(x)$ is the ball of radius $r$ around $x$. In general the local dimension may not exist but it exists in the cases which will interest us. We will work with measures of intermediate dimension, meaning that $0<D(\mu, x)<1$ at $\mu$-a.e. $x$. This rules out Lebesgue component, and also any zero-dimensional component, including atoms.

In this work we consider probability measures which are invariant under the maps $T_{a}:[0,1] \rightarrow[0,1]$ defined for integers $a \geq 2$ by

$$
T_{a} x=a x \bmod 1,
$$

i.e. measures such that $T_{a} \mu=\mu$. We sometimes view $T_{a}$ as a map of the torus $\mathbb{R} / \mathbb{Z}$. We note that there is a very rich supply of $T_{a}$-invariant measures, including some self-similar measures but also many (most) which are not.

For $T_{a}$-invariant measures most work to date has focused on their behavior for maps which are related to the group structure of $\mathbb{R} / \mathbb{Z}$, i.e. when $f$ is one of the maps $T_{b}$ (an endomorphism of $\mathbb{R} / \mathbb{Z}$ ) or translation by an element of $\mathbb{R} / \mathbb{Z}$. The principal result for endomorphisms is the measure rigidity theorem of Rudolph [18] and Johnson [10]. Write $a \sim b$ if $a, b$ are powers of a common integer, i.e. $a=c^{k}$ and $b=c^{m}$ for some $c, k, m \in \mathbb{N}$, and otherwise write $a \nsim b$. The Rudolph-Johnson Theorem states that if $\mu$ is a $T_{a}$-invariant measure whose ergodic components all have entropy strictly between 0 and $\log a$, then it is not preserved by $T_{b}$ for any $b \nsim a$. The result can be slightly improved, using a later result of Rudolph-Johnson [12], to conclude that $\mu \perp v$ for every $T_{b}$-invariant measure $v$ of intermediate dimension. To date this is essentially the best result towards Furstenberg's $\times 2, \times 3$ conjecture, which predicts that there should be no non-atomic measures except Lebesgue which are jointly invariant under $T_{a}$ and $T_{b}$ for $a \nsim b$. For a survey of related algebraic conjectures and results see [14]. There are also strong rigidity results for smooth actions of $\mathbb{Z}^{d}$ and $\mathbb{R}^{d}$ : see e.g. Kalinin, Katok and Hertz [13].

For translations we are aware only of the work of Host [8]. Define $\mu$ to be conservative for a subgroup $\Lambda \subseteq \mathbb{R} / \mathbb{Z}$ if for every set $A$ with $\mu(A)>0$ there is some $0 \neq r \in \Lambda$ such that $\mu(A \cap(A+r))>0$. For the groups $\Lambda=\mathbb{Z}[1 / b]$ of $b$-adic rationals when $\operatorname{gcd}(a, b)=1$, or for the cyclic subgroup generated by an element $r \in \mathbb{R} / \mathbb{Z}$ such that $\left\{T_{a}^{n} r\right\}_{n \in \mathbb{N}}$ is dense in $\mathbb{R} / \mathbb{Z}$, Host showed, using methods of harmonic analysis, that the only conservative $T_{a}$-invariant measure is Lebesgue measure. Note that these results do not require any assumption about the dimension of the measure, but also they do not directly relate to our question, since they do not say anything about preservation of $\mu$ under a particular rotation.

In another direction, the question we are interested in has been studied in the fractal geometry literature for rather general functions $f$, e.g. $C^{1}$ or bi-Lipschitz, but for restricted classes of measures such as self-similar measures on attractors of iterated function 
systems. In situations like these it has been shown that measures arising from different parameters are mutually singular, and cannot be easily deformed into each other (see for example [3]). Related results for certain classes of Cantor sets are known (see for instance $[2,1,20])$.

\subsection{Statement of results}

Write $\mu \sim v$ and $\mu \perp v$ to indicate that the measures are equivalent or singular, respectively, and let $\left.\mu\right|_{E}$ denote the restricted measure $\left.\mu\right|_{E}(A)=\mu(A \cap E)$. Thus $\mu, v$ are non-singular if and only if $\left.\left.\mu\right|_{E} \sim \nu\right|_{E}$ for some set $E$ with $\nu(E)>0$.

Theorem 1.1. Let $\mu$ be a $T_{a}$-ergodic measure of intermediate dimension. Then there exists $n \in \mathbb{N}$ such that if $f \in \operatorname{diff}(\mathbb{R})$ and $\left.\left.(f \mu)\right|_{E} \sim \mu\right|_{E}$, then

$$
f^{\prime}(x) \in\left\{ \pm a^{k / n}: k \in \mathbb{Z}\right\} \quad \text { for } \mu \text {-a.e. } x \in f^{-1} E \text {. }
$$

More generally, if $v$ is another $T_{a}$-ergodic measure and $\left.\left.(f \mu)\right|_{E} \sim \nu\right|_{E}$ then there exists $t \in \mathbb{R}$ such that

$$
f^{\prime}(x) \in\left\{ \pm t \cdot a^{k / n}: k \in \mathbb{Z}\right\} \quad \text { for } \mu \text {-a.e. } x \in f^{-1} E .
$$

While we have stated the result for diffeomorphisms of $\mathbb{R}$, the result is of a local nature and immediately applies to partially defined or piecewise diffeomorphisms.

Theorem 1.1 is close to optimal. One clearly cannot hope to get information about $f$ except on the support of $\left.\mu\right|_{E}$. On the other hand, a $T_{a}$-invariant measure $\mu$ is also invariant for $T_{a^{n}}$ for every $n \in \mathbb{N}$ and sometimes also for $n=1 / m$ when $a^{1 / m} \in \mathbb{N}$. Thus one cannot expect that some intrinsic property of $\mu$ will encode $a$, and the best one can hope for is a power of $a$. The ergodicity assumption is necessary also: for example fix a $T_{2}$ invariant measure $\mu$ of intermediate dimension and form $v=\frac{1}{2} \mu+\frac{1}{2} T_{3} \mu$, which is also $T_{2}$-invariant. It is easy to see that there is a piecewise linear map $f$ with $f v, v$ nonsingular, and with slopes 2 and 3 on sets of positive $v$-measure.

We do not know whether it can happen in the theorem that $a^{1 / n}$ is not an integer. We also do not know whether a version of the theorem is true under Lipschitz (rather than differentiability) conditions on $f$.

Under mild additional assumptions, Theorem 1.1 implies that very few maps can preserve a $T_{a}$-ergodic measure of intermediate dimension.

Corollary 1.2. Let $\mu$ and $n$ be as in the theorem. Then every piecewise analytic map of $[0,1]$ which preserves $\mu$ is piecewise linear on an open set $U$ containing the support of $\mu$ and on $U$ has slopes of the form $\pm a^{k / n}, k \in \mathbb{N}$.

Corollary 1.3. If $\mu$ is a $T_{a}$-ergodic measure on $\mathbb{R} / \mathbb{Z}$ which has intermediate dimension and is globally supported, then every $C^{1}$-map which preserves $\mu$ has the form $T_{a^{\prime}}$ for an integer $a^{\prime} \sim a$. 
Easy examples show that there can be piecewise linear maps other than $T_{a}$ which preserve $\mu$, for example one can easily construct them by hand for the uniform measure on the middle third Cantor set (i.e. Hausdorff measure at the appropriate dimension, normalized to mass 1 ), which is $T_{3}$-invariant.

As a special case of the above we recover the Rudolph-Johnson Theorem [18, 10]. One may speculate that Theorem 1.1 holds without the entropy assumption, but proving this would imply the full $\times 2, \times 3$ conjecture.

Our methods also allow us to generalize the Rudolph-Johnson Theorem in other ways:

Theorem 1.4. If $a \nsim b$ and $\mu, v$ are respectively $T_{a}$-and $T_{b}$-ergodic measures of intermediate dimension, then $f \mu \perp v$ for every $f \in \operatorname{diff}^{2}(\mathbb{R})$.

We can eliminate the ergodicity and regularity assumptions under some (rather weak) additional hypotheses, for example no ergodicity is needed if $f$ is affine, or when $f \in$ $\operatorname{diff}(\mathbb{R})$ but the ergodic components of $v$ under $T_{b}$ do not have spectrum of the form $n / \log a$.

An interesting consequence of the theorem above is that the measure rigidity phenomenon in the Rudolph-Johnson Theorem is not a consequence of properties of the abelian action generated by $T_{a}$ and $T_{b}$, but rather of the smooth conjugacy classes of the individual maps $T_{a}, T_{b}$ :

Corollary 1.5. Let $a \nsim b$ and let $f, g$ be self-maps of $\mathbb{R} / \mathbb{Z}$ which are (separately) $C^{2}$-conjugate to $T_{a}, T_{b}$, respectively. Then there is no measure of positive Hausdorff dimension which is ergodic for both $f$ and $g$, except possibly one which is equivalent to Lebesgue, and this occurs precisely when the conjugating maps differ by a rotation.

Note that for $f, g$ as above there will generally be no invariant measures at all, but it is hard to verify this for any particular pair of conjugates. It is known that if $f, g$ commute then they are simultaneously $C^{0}$-conjugate to $T_{a}, T_{b}$ [11], and then Corollary 1.5 follows from the Rudolph-Johnson Theorem.

After this paper was completed P. Shmerkin suggested another approach which proves Theorem 1.4 for $f \in \operatorname{diff}(\mathbb{R})$ and non-ergodic measures, but which does not give any version of Theorem 1.1. This will appear elsewhere.

\subsection{Methods}

To arrive at these results we study measures on $\mathbb{R}^{d}$ through the dynamics of the 1parameter, measure-valued family obtained by "zooming in" on typical points for the measure. These families are called sceneries, and for the measures we are considering they behave like generic orbits in an appropriate dynamical system. Many variants of the notion of a scenery have appeared in the fractal geometry literature (see e.g. Bedford and Fisher [1]), and have been used as a technical tool in the study of $T_{m}$-invariant measures, in disguised form in Furstenberg's paper [4] and more recently in [7]. Our definition of the scenery flow follows that of Gavish [5]. A systematic study of this notion and related ones can be found in [6]. 
Briefly, we show that for a $T_{a}$-invariant measure the sceneries equidistribute for an ergodic flow whose pure point spectrum contains a rational multiple of $1 / \log a$, and the remaining spectrum comes from the original dynamics of $T_{a}$. Since these flows are associated to measures in a geometric way, they are invariants of the measure under the application of differentiable, locally bijective maps. Furthermore, the flows derived at different points of a $T_{a}$-invariant measure may exhibit different phases with respect to eigenvalues of the form $n / \log a$, and applying a smooth map shifts the phase by the logarithm of the derivative. The behavior of these phases underlies the proof of Theorems 1.1 and 1.4 .

This method of proof gives a concrete necessary condition for a measure $\mu$ to be a smooth image of a $T_{a}$-invariant measure, namely the spectrum of the associated flow must contain a rational multiple of $\log a$. In a sense, this explains how $\mu$ "encodes" the arithmetic class of the dynamics which generated it.

\subsection{Related questions}

We end this introduction with some open questions. Let us begin by pointing out a connection between Theorem 1.4 and another conjecture of Furstenberg [4]: If $X, Y \subseteq[0,1]$ are closed and invariant, respectively, under $T_{a}$ and $T_{b}$ for $a \nsim b$, then for every affine $\operatorname{map} f(x)=u x+v$,

$$
\operatorname{dim}(X \cap f Y) \leq \max \{0, \operatorname{dim} X+\operatorname{dim} Y-1\} .
$$

This says that all affine images of $Y$ should intersect $X$ in as small a set as possible. Theorem 1.4 gives an analog of this for measures, though of course singularity of measures implies nothing about the intersection of their topological supports. On the other hand, note that Theorem 1.4 has content even when both $\mu, v$ are globally supported.

Returning to the $\times 2, \times 3$ conjecture, the topological version was proved by Furstenberg with no entropy assumptions: any closed infinite subset of $[0,1]$ which is invariant under $T_{a}, T_{b}$ for $a \nsim b$ is the entire interval. One may similarly ask for topological versions of our results:

Problem 1.6. Suppose $A \subseteq[0,1]$ is an infinite, proper closed $T_{a}$-invariant subset. If $f \in \operatorname{diff}^{1}(\mathbb{R})$ preserves $A$, must $\left|f^{\prime}(x)\right|$ be in $\left\{a^{r}: r \in \mathbb{Q}\right\}$ for all non-isolated $x \in A$ ?

Problem 1.7. Let $a \nsim b$ and let $f, g$ be maps of $\mathbb{R} / \mathbb{Z}$ which are (separately) conjugate, respectively, to $T_{a}, T_{b}$. If the conjugating maps are sufficiently smooth, can we conclude that there are no infinite, closed proper subsets of $\mathbb{R} / \mathbb{Z}$ which are jointly $f$-and $g$-invariant?

We do not have answers except when for some $s>0$ the $s$-dimensional Hausdorff measure is positive and finite on $A$. Then one can apply our results to this measure.

In another direction, there is a strengthening of the Rudolph-Johnson Theorem due Host [8], which asserts that for $\operatorname{gcd}(a, b)=1$, if $\mu$ is $T_{a}$-invariant of intermediate dimension, then $\mu$-a.e. point $x$ equidistributes for Lebesgue measure under the action of $T_{b}$ (in this case $x$ is said to be normal in base $b$ ). It is natural to ask whether the same is true 
when the measure is distorted by a nice enough map. Let us formulate this question in the simplest and most plausible case:

Problem 1.8. Let $\mu$ be a $T_{a}$-invariant measure and of intermediate dimension, and $\operatorname{gcd}(a, b)=1$. If $f(x)=u x+v, u \neq 0$, is $f \mu$-a.e. point normal in base $b$ ?

Finally, it is very likely that analogs of Theorems 1.1 and 1.4 hold for more general interval maps and in higher-dimensional settings, for instance for measures on the torus which are invariant under hyperbolic automorphisms under suitable assumptions. However, in neither case does it appear that our methods apply directly.

\subsection{Organization}

In Section 2 we define the scenery flow in more detail, state without proof our results about its spectral properties, and deduce the main results. The remaining proofs are given in Section 3. We assume familiarity with basic notions in ergodic theory, recalling some definitions as we go; for an introduction see [19]. For background on geometric measure theory see [16].

\section{Main elements of the proofs}

In this section we give our main definitions and technical results, and derive the main theorems from them. The remaining proofs are provided in the next section.

\subsection{The scenery flow}

Let $\mathcal{M}=\mathcal{M}_{d}$ denote the space of Radon measures on $\mathbb{R}^{d}$, endowed with the weak topology. We use the term measure for Radon measures on $\mathbb{R}^{d}$, and denote measures by $\mu, v, \sigma, \tau$ etc. We reserve the term distribution for Borel probability measures on $\mathcal{M}$, which we denote by $P, Q, R$ etc. The space of distributions carries a measurable structure defined by declaring the map $\mu \mapsto \mu(A)$ to be measurable for all Borel sets $A \subseteq \mathcal{M}$. Write $\lambda$ for Lebesgue measure and $\delta_{z}$ for the point mass at $z$, which is a measure when $z \in \mathbb{R}^{d}$ and a distribution when $z \in \mathcal{M}$. Let supp $\mu$ denote the topological support of a measure, that is, the complement of the union of all open sets of $\mu$-measure zero. We write $\sim$ for equivalence of measures or distributions, and also write $z \sim \mu$ to indicate that $z$ is distributed according to $\mu$; the intended meaning will be clear from the context.

For $x \in \mathbb{R}^{d}$ let $U_{x}: \mathbb{R}^{d} \rightarrow \mathbb{R}^{d}$ denote the translation map

$$
U_{x}(y)=y-x,
$$

and for $t \in \mathbb{R}$ let $S_{t}: \mathbb{R}^{d} \rightarrow \mathbb{R}^{d}$ denote the scaling map

$$
S_{t}(x)=e^{t} x .
$$

Note the exponential time scale, which makes $S=\left(S_{t}\right)_{t \in \mathbb{R}}$ into an action of the additive group $\mathbb{R}$ on $\mathbb{R}^{d}$. These operations induce maps on $\mathcal{M}$ : for $\mu \in \mathcal{M}$ we have $U_{x} \mu(A)=$ $\mu(A+x)$ and $S_{t} \mu(A)=\mu\left(e^{-t} A\right)$. 
Let $\mu \mapsto \mu^{\square}$ denote the operation of normalizing a measure to have unit mass on $[-1,1]^{d}$ and restricting it to this cube, i.e.

$$
\mu^{\square}=\left.\frac{1}{\mu\left([-1,1]^{d}\right)} \mu\right|_{[-1,1]^{d} .}
$$

Let

$$
\mathcal{M}^{\square}=\left\{\text { Probability measures on } \mathbb{R} \text { supported on }[-1,1]^{d}\right\}
$$

and define $S_{t}^{\square}: \mathcal{M}^{\square} \rightarrow \mathcal{M}^{\square}$ by

$$
S_{t}^{\square} \mu=\left(S_{t} \mu\right)^{\square}
$$

so that $S^{\square}=\left(S_{t}^{\square}\right)_{t \geq 0}$ is a semigroup acting on the set of $\mu \in \mathcal{M}^{\square}$ with $0 \in \operatorname{supp} \mu$. This is a Borel subset of $\mathcal{M}$, and the action is Borel, though not continuous (it is discontinuous at measures which give positive mass to the boundary of $\left.[-1,1]^{d}\right)$.

Definition 2.1. Let $\mu \in \mathcal{M}$ and $x \in \operatorname{supp} \mu$. The scenery of $\mu$ at $x$ is the orbit of $U_{x} \mu$ under $S^{\square}$, i.e. the one-parameter family

$$
\mu_{x, t}=S_{t}^{\square}\left(U_{x} \mu\right), \quad t \in \mathbb{R}^{+} .
$$

In other words, the scenery is what one sees when "zooming in" to $\mu$ at $x$, restricting and normalizing the measure as we go.

In order to discuss the limiting behavior of the scenery, note that $\mathcal{M}^{\square}$ may be identified with the weak-* compact set of probability measures on $[-1,1]^{d}$. Thus we may speak of convergence of distributions on $\mathcal{M}^{\square}$.

Definition 2.2. A measure $\mu \in \mathcal{M}$ generates a distribution $P$ at $x \in \operatorname{supp} \mu$ if the scenery $\left(\mu_{x, t}\right)_{t \geq 0}$ equidistributes for $P$, that is, if the uniform measure on the path $\left(\mu_{x, t}\right)_{0 \leq t \leq T}$ converges weak-* to $P$ as $T \rightarrow \infty$. Equivalently, for every $f \in C\left(\mathcal{M}^{\square}\right)$,

$$
\lim _{T \rightarrow \infty} \frac{1}{T} \int_{0}^{T} f\left(\mu_{x, t}\right) d t=\int f d P .
$$

In this case $\left(\mathcal{M}^{\square}, P, S^{\square}\right)$ is called the scenery flow of $\mu$ at $x$.

For a discussion of the properties of distributions generated in this way see [6]. We mention a few basic facts. First, if there is a positive $\mu$-measure of points at which $\mu$ generates some distribution (which may vary from point to point), then $\mu$-a.e. one of the distributions is $S^{\square}$-invariant. ${ }^{1}$ Second, standard density arguments show that if $v \ll \mu$ then a $\nu$-typical point generates a distribution for $v$ if and only if it does for $\mu$, and in this case the distributions are the same. This applies in particular when $v=\left.\mu\right|_{A}$.

The following simple observation is a key ingredient in our arguments. Let $\operatorname{diff}_{+}(\mathbb{R})$ $\subseteq \operatorname{diff}(\mathbb{R})$ denote the subgroup of orientation preserving maps.

\footnotetext{
1 Note that $S^{\square}$ acts discontinuously, so this is not a complete triviality.
} 
Lemma 2.3. Let $\mu \in \mathcal{M}(\mathbb{R}), x \in \operatorname{supp} \mu$ and $f \in \operatorname{diff}_{+}^{1}(\mathbb{R})$. Then after a time-shift of $s=\log f^{\prime}(x)$ the sceneries $\mu_{x, t}$ and $(f \mu)_{f(x), t}$ are asymptotic, i.e.

$$
\lim _{t \rightarrow \infty}\left(\mu_{x, t}-(f \mu)_{f(x), t-s}\right)=0 \quad\left(\text { weak-*) }{ }^{*}\right.
$$

In particular $\mu$ generates $P$ at $x$ if and only if $f \mu$ generates $P$ at $f(x)$.

The proof is immediate from the fact that, locally, $f$ acts like $S_{s}$ near $x$.

The assumption that $f$ preserve orientation is necessary for the conclusion that the scenery flows are the same, but if $f$ is orientation reversing then the scenery flows are isomorphic to measure preserving flows by way of the map induced on $\mathcal{M}^{\square}$ from $x \mapsto$ $-x$. We omit the details. More generally, a similar lemma holds in $\mathbb{R}^{d}$ but then one must also account for skewing of the image.

\subsection{The scenery flow of $T_{a}$-invariant measures and its spectral properties}

Next we describe the scenery flow of a $T_{a}$-invariant measure and, more generally, products of such measures. Recall that the diagonal action of $T_{a}$ on $[0,1]^{d}$ is given by $T_{a}(x)=$ $\left(T_{a} x_{1}, \ldots, T_{a} x_{d}\right)$. A product of $T_{a}$-invariant measures is invariant under the diagonal action.

We require two more standard constructions, which we recall briefly. First, the natural extension of an ergodic system $(\Omega, \nu, T)$ is an invertible ergodic system $(\widetilde{\Omega}, \widetilde{v}, \widetilde{T})$ factoring onto $(\Omega, v, T)$ and characterized by the property that every factor map from an invertible system to $(\Omega, v, T)$ factors through $(\widetilde{\Omega}, \widetilde{\nu}, \widetilde{T})$. The natural extension may be realized as the inverse limit of the diagram of factor maps

$$
\cdots \rightarrow(\Omega, v, T) \stackrel{T}{\rightarrow}(\Omega, v, T) \stackrel{T}{\rightarrow}(\Omega, v, T) .
$$

See also Section 3.

Second, the $t_{0}$-suspension of the discrete time system $(\Omega, \nu, T)$ is the flow defined on $\Omega \times\left[0, t_{0}\right]$ by

$$
T_{t}(\omega, s)=\left(T^{\left[(s+t) / t_{0}\right]} \omega,\left\{\frac{s+t}{t_{0}}\right\} t_{0}\right)
$$

where $[r]$ and $\{r\}$ are the integer and fractional parts of $r$, respectively. This flow preserves the product measure $v \times\left.\frac{1}{t_{0}} \lambda\right|_{\left[0, t_{0}\right)}$.

Proposition 2.4. Let $\mu_{1}, \ldots, \mu_{d}$ be $T_{a}$-invariant measures and $\mu=\times_{i=1}^{d} \mu_{i}$. Then $\mu$ generates an $S^{\square}$-ergodic distribution $P_{x}$ at a.e. point $x$, and the system $\left(\mathcal{M}^{\square}, P_{x}, S^{\square}\right)$ arises as a factor of the $\log b$-suspension of the ergodic component $\mu^{(x)}$ of $x$ in $\left([0,1]^{d}, \mu, T_{a}\right)$. In particular $P_{x}$ depends only on the ergodic component $\mu^{(x)}$ of $x$. Furthermore, if $\mu^{(x)}$ has intermediate dimension then $P_{x}$ is supported on measures of intermediate dimension.

The construction and analysis are carried out in detail in Sections 3.1-3.4. 
Write $e(t)=\exp (2 \pi i t)$. Recall that $\alpha \in \mathbb{R}$ is an eigenvalue of an ergodic measure preserving system $(\Omega, \mathcal{B}, v, T)$ if there is a complex function $\varphi \in L^{2}$ such that $\varphi \circ T=$ $e(\alpha) \varphi$, and $\alpha$ is an eigenvalue of a measure preserving flow $\left(\Omega, \mathcal{B}, v,\left(T_{t}\right)_{t \in \mathbb{R}}\right)$ if there is a function $\varphi \in L^{2}$ such that $\varphi \circ T_{t}=e(\alpha t) \varphi$ for all $t \in \mathbb{R}$. Such $\varphi$ are called eigenfunctions, and for ergodic transformations and flows they a.s. have constant modulus, which we shall always assume has been normalized to 1 . We denote the set of eigenvalues by $\Sigma$, with subscripts to indicate the system in question.

Theorem 2.5. Let $\mu$ be $T_{a}$-invariant with intermediate entropy. Let $\Sigma_{\mu^{(x)}}$ denote the spectrum of the ergodic component $\mu^{(x)}$ of $\left([0,1], \mu, T_{a}\right)$ to which $x$ belongs. Let $P_{x}$ be the distribution generated at $x$, and $\Sigma_{P_{x}}$ the spectrum of $\left(\mathcal{M}^{\square}, P_{x}, S^{\square}\right)$. Then there is an $n \in \mathbb{N}$ such that

$$
\frac{n}{\log a} \mathbb{Z} \subseteq \Sigma_{P_{x}} \subseteq \frac{1}{\log a} \Sigma_{\mu^{(x)}} \cup \frac{n}{\log a} \mathbb{Z}
$$

The proof is given in Section 3.6. Note that in the theorem one cannot assume that $n=1$

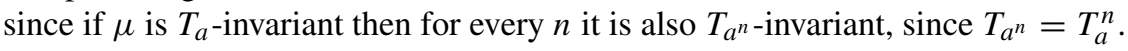

Let us show how this theorem implies one of our main results:

Proof of Theorem 1.4 under spectral assumptions. Suppose $f \in \operatorname{diff}^{1}$ and $\mu, v$ are respectively $T_{a}, T_{b}$-invariant, have intermediate entropy, and the ergodic components of $v$ do not have pure point spectrum of the form $n / \log a, n \in \mathbb{Z}$. By the theorem above the scenery flows generated a.e. by $\mu$ have pure point spectrum of this form. If $f \mu \not \perp v$ then by Lemma 2.3 , with positive $\mu$-probability, the scenery flow generated by $\mu$ at $x$ is isomorphic to the one generated by $v$ at $f(x)$. These possibilities are incompatible.

\subsection{The distribution of phases}

More refined information can be obtained from the distribution of phases of the eigenfunctions of the scenery flow. That is, for a $T_{a}$-ergodic measure and typical points $x, y$, we may consider the sceneries at $x$ and $y$ and compare the relative phase of the eigenfunctions corresponding to $\alpha=k / \log a$. There is a technical problem with this idea, since the eigenfunctions are defined only a.e. for the scenery flow distribution, and not necessarily on any of the measures of the scenery itself. To overcome this, one can consider the joining of the scenery flow generated at the two points.

Recall that a joining of $S^{\square}$-invariant distributions $P_{1}, P_{2}$ is a distribution $P$ on $\mathcal{M}^{\square} \times \mathcal{M}^{\square}$ which projects to $P_{i}$ on the $i$-th coordinate, and which is invariant under the diagonal flow $S^{\square}$ given by $S_{t}^{\square}(\mu, v)=\left(S_{t}^{\square} \mu, S_{t}^{\square} v\right)$. When $P_{1}, P_{2}$ are ergodic, the ergodic components of a joining of $P_{1}, P_{2}$ are also joinings of $P_{1}, P_{2}$. A $P$-joining is a joining of $P$ with itself.

Let $\mu$ be a measure generating an ergodic distribution $P_{x}$ at $x$. Note that there is a bijective correspondence between pairs $(\sigma, \tau) \in \mathcal{M}_{1}^{\square} \times \mathcal{M}_{1}^{\square}$ and product measures $\sigma \times \tau \in \mathcal{M}_{2}^{\square}$, and that the set of product measures is closed in $\mathcal{M}^{\square} \times \mathcal{M}^{\square}$. Therefore the accumulation points of the sceneries of $\mu \times \mu$ are product measures, and if $\mu \times \mu$ generates a scenery flow it is supported on product measures. Furthermore, if $\mu \times \mu$ generates 
a scenery flow $P_{x, y}$ at $(x, y)$, one may verify that, making the identification between product measures and pairs, $P_{x, y}$ is a joining of the scenery flows $P_{x}, P_{y}$ generated by $\mu$ at $x$ and $y$. Note that by Proposition 2.4, if $\mu$ is $T_{a}$-invariant then $\mu \times \mu$ indeed generates sceneries a.e.

Let $P$ be an $S^{\square}$-invariant and ergodic distribution with an eigenvalue $\alpha$ and corresponding eigenfunction $\varphi$. Then the function $p_{\alpha}: \mathcal{M}^{\square} \times \mathcal{M}^{\square} \rightarrow \mathbb{C}$ defined by

$$
p_{\alpha}(\sigma, \tau)=\frac{\varphi(\sigma)}{\varphi(\tau)}
$$

is a.e. invariant on any ergodic $P$-joining, since

$$
p_{\alpha}\left(S_{t}^{\square} \sigma, S_{t}^{\square} \tau\right)=\frac{\varphi\left(S_{t}^{\square} \sigma\right)}{\varphi\left(S_{t}^{\square} \tau\right)}=\frac{e(\alpha t) \varphi(\sigma)}{e(\alpha t) \varphi(\tau)}=p_{\alpha}(\sigma, \tau) .
$$

Therefore if $R$ is a $P$-joining then $p_{\alpha}$ is constant on a.e. ergodic component of $R$, and if $R$ is ergodic then we may define

$$
p_{\alpha}(R)=R \text {-a.s. value of } p_{\alpha}(\cdot, \cdot) .
$$

Let $\mu$ generate some distribution $P$ at a.e. point and assume that $\mu \times \mu$ generates an ergodic $P$-joining $P_{x, y}$ at $\mu \times \mu$-a.e. $(x, y)$. Fix a $\mu$-typical $x_{0}$ so that $P_{x_{0}, y}$ is defined for $\mu$-a.e. $y$ and for such $y$ let

$$
p_{\alpha}\left(\mu, x_{0}, y\right)=p_{\alpha}\left(P_{x_{0}, y}\right) .
$$

Definition 2.6. Let $\mu$ be a measure which a.e. generates $P$, and such that $\mu \times \mu$ generates an ergodic distribution at a.e. point. Let $\alpha \in \Sigma_{P}$. For a $\mu$-typical point $x_{0}$ the phase measure $\theta_{\alpha}=\theta_{\alpha}\left(\mu, x_{0}\right)$ is the push-forward of $\mu$ under the map $y \mapsto p_{\alpha}\left(\mu, x_{0}, y\right)$.

Under the further assumption that $\mu \times \mu \times \mu$ generates an ergodic distribution at a.e. point, the dependence of the phase measure on $x_{0}$ is very mild. Indeed, if we choose another point $x_{1}$ then, by considering the threefold joining $Q$ generated by $\mu \times \mu \times \mu$ at $\left(x_{0}, y, x_{1}\right)$ we find that, writing $\left(\sigma_{1}, \sigma_{2}, \sigma_{3}\right)$ for a $Q$-typical element,

$$
p_{\alpha}\left(\mu, x_{1}, y\right)=\frac{\varphi\left(\sigma_{3}\right)}{\varphi\left(\sigma_{2}\right)}=\frac{\varphi\left(\sigma_{3}\right)}{\varphi\left(\sigma_{1}\right)} \cdot \frac{\varphi\left(\sigma_{1}\right)}{\varphi\left(\sigma_{2}\right)}=c \cdot p_{\alpha}\left(\mu, x_{0}, y\right),
$$

where $c=p_{\alpha}\left(\mu, x_{1}, x_{0}\right)$ does not depend on $y$. Thus $p_{\alpha}\left(\mu, x_{0}, \cdot\right)$ depends on $x_{0}$ only up to a rotation, and the measures $\theta_{\alpha}\left(\mu, x_{0}\right)$ and $\theta_{\alpha}\left(\mu, x_{1}\right)$ are rotations of one another. Since we shall be interested in properties which are independent of rotation, we often suppress the dependence on $x_{0}$ and write $\theta_{\alpha}(\mu)$.

Theorem 2.7. Let $\mu$ be a $T_{a}$-invariant measure of intermediate entropy whose ergodic components a.s. generate the same distribution P. Let $\alpha \in \Sigma_{P}$ and $\theta_{\alpha}=\theta_{\alpha}(\mu)$. Then

(i) If $\alpha \in \Sigma_{P} \backslash(1 / \log a) \mathbb{Q}$ then $\theta_{\alpha}$ is Lebesgue measure.

(ii) If $\alpha \in \Sigma_{P} \cap(1 / \log a) \mathbb{Q}$ then $\theta_{\alpha}$ is singular with respect to Lebesgue measure.

(iii) If $\alpha \in \Sigma_{P} \cap(1 / \log a) \mathbb{Z}$ and $\mu$ is ergodic then $\theta_{\alpha}$ consists of a single atom.

This is proved in Section 3.7. 
We do not know how large the phase measure can be for non-ergodic $\mu$. With our methods we can go a little further and show that $\underline{\operatorname{dim}} \theta_{\alpha}(\mu) \leq 1-\operatorname{dim} \mu$, where $\underline{\operatorname{dim}}$ is the lower Hausdorff dimension of $\mu$. On the other hand, starting with a $T_{a}$-ergodic measure $\mu$ with $\alpha=n / \log a$ in the spectrum of the scenery flow, the measure $v=\sum_{b=1}^{\infty} 2^{-b} T_{b} \mu$ is again $T_{a}$-invariant and its phase measure consists of atoms at $e(\log b / \log a), b \in \mathbb{N}$. We suspect that the phase measure is always atomic or at least of dimension zero, but we have not resolved this.

We next examine how the phase distribution changes when a smooth map is applied to a measure. First, suppose that $\mu$ is a measure satisfying the conditions in Definition 2.6 and the discussion following it. In particular for $\mu \times \mu$-a.e. $(x, y)$ the scenery $\left(\mu_{x, t} \times\right.$ $\left.\mu_{y, t}\right)_{t \geq 0}$ equidistributes for some $P$-joining $P_{x, y}$. Now let $s \in \mathbb{R}$ and consider the family $\left(\mu_{x, t} \times \mu_{y, t+s}\right)_{t \geq 0}$, in which we have shifted the second component by $S_{s}^{\square}$. This family equidistributes for the $P$-joining $Q=\left(\mathrm{id} \times S_{s}^{\square}\right) P_{x, y}$ obtained as the push-forward of $P_{x, y}$ through the map $(\sigma, \tau) \mapsto\left(\sigma, S_{S}^{\square} \tau\right){ }^{2}$ For $\alpha \in \Sigma_{P}$ and corresponding eigenfunction $\varphi$, let $(\sigma, \tau)$ be a $P_{x, y}$-typical pair such that $\left(\sigma, S_{s}^{\square} \tau\right)$ is $Q$-typical. Then

$$
p_{\alpha}(Q)=p_{\alpha}\left(\sigma, S_{s}^{\square} \tau\right)=\frac{\varphi(\sigma)}{e(\alpha s) \varphi(\tau)}=e(-\alpha s) \cdot p_{\alpha}(\sigma, \tau)=e(-\alpha s) \cdot p_{\alpha}\left(P_{x, y}\right) .
$$

Together with Lemma 2.3, this leads to the following result:

Proposition 2.8. Let $\mu$ be a $T_{a}$-invariant measure of intermediate dimension which a.e. generates a distribution $P$, let $\alpha \in \Sigma_{P}$ and let $f \in \operatorname{diff}^{1}(\mathbb{R})$. Then $\theta_{\alpha}(f \mu)$ is well defined, and, fixing a $\mu$-typical $x_{0}$, is given up to rotation by

$$
\theta_{\alpha}(f \mu)=\int \delta_{e\left(-\alpha \log f^{\prime}(y)\right) \cdot p_{\alpha}\left(P_{x_{0}, y}\right)} d \mu(y) .
$$

The discussion above proves the proposition when $f$ preserves orientation. An obvious modification of the statement and proof is needed when $f$ is orientation reversing. See the remark after Lemma 2.3.

\subsection{Proof of the main results}

Proof of Theorem 1.1. Let $\mu, v$ be $T_{a}$-ergodic measures of intermediate dimension. Suppose $f \in \operatorname{diff}^{1}(\mathbb{R})$ and $\left.\left.f \mu\right|_{E} \sim v\right|_{E}$ for some set $E$ with $v(E)>0$. Then $\theta_{\alpha}\left(\left.f \mu\right|_{E}\right)$ and $\theta_{\alpha}\left(\left.\nu\right|_{E}\right)$ are equivalent for every $\alpha \in \Sigma_{P}$. Choosing $n \in \mathbb{N}$ and $\alpha=n / \log a \in \Sigma_{P}$, as we may by Theorem 2.5, it follows from Theorem 2.7 that $\theta_{\alpha}(v)$ is a point mass. Therefore $\theta_{\alpha}\left(\left.f \mu\right|_{E}\right)$ is a point mass, and by Proposition 2.8 this implies that $e\left(\alpha \cdot \log f^{\prime}(\cdot)\right)$ is $\mu$-a.s. constant on $f^{-1} E$, giving the result.

In the case $v=\mu$ we have $\left.\left.f \mu\right|_{E} \ll \mu\right|_{E}$ so $\theta_{\alpha}\left(\left.f \mu\right|_{E}\right) \ll \theta_{\alpha}(\mu)$, and since both consist of a single atom we have equality. Hence $e\left(\alpha \cdot \log f^{\prime}(\cdot)\right)=1$ at $\mu$-a.e. point of $f^{-1} E$, so $\left.f^{\prime}\right|_{f^{-1} E}$ is $\mu$-a.e. an integer power of $a^{1 / n}$.

Before proving the next theorems we require one more technical result:

\footnotetext{
2 This requires a short argument since $S^{\square}$ is not continuous, but we omit it.
} 
Proposition 2.9. Let $\mu$ be a $T_{a}$-invariant measure of intermediate entropy generating $P$, and $\alpha \in \Sigma_{P} \cap(1 / \log a) \mathbb{Z}$. Suppose one of the following holds:

(i) $\mu$ is ergodic and $f \in \operatorname{diff}^{2}(\mathbb{R})$,

(ii) $f$ is affine.

Then $\theta_{\alpha}(f \mu)$ is singular with respect to Lebesgue measure.

Proof. In the first case $\theta_{\lambda}\left(\mu, x_{0}\right)$ consists of a single atom (Theorem 2.7), and $p_{\lambda}\left(x_{0}, \cdot\right)$ is independent of $y$. Then by Proposition 2.8, up to rotation, $\theta_{\alpha}(f \mu)$ is the image of $\mu$ under $g: x \mapsto e\left(f^{\prime}(x)\right)$. Since $f \in C^{2}$ we have $f^{\prime} \in C^{1}$ and in particular $f^{\prime}$, and hence $g$, is Lipschitz. Since $\mu$ has intermediate dimension, it is singular with respect to Lebesgue measure, so this is also true of $g \mu$, as desired.

In the second case $f(x)=u x+v$ and we need only consider the case $u \neq 0$. Since $f^{\prime}(x)$ does not depend on $x$, we find by Proposition 2.8 that $\theta_{\alpha}(f \mu)$ is a rotation of $\theta_{\alpha}(\mu)$ by $e(\log u)$, and so, since $\theta_{\lambda}$ is singular by Theorem 2.7, so is $\theta_{\alpha}(f \mu)$.

Proof of Theorem 1.4 and variants. Let $\mu$ be a $T_{a}$-invariant measure and $v$ a $T_{b}$-invariant measure, both of intermediate entropy. Suppose that $f \in \operatorname{diff}^{2}(\mathbb{R})$ and that $\left.\left.(f \mu)\right|_{E} \sim \nu\right|_{E}$ for some $E$ with $v(E)>0$. Then $\theta_{\alpha}\left(\left.f \mu\right|_{E}\right)$ is equivalent to $\theta_{\alpha}\left(\left.\nu\right|_{E}\right)$ for all $\alpha$, and it suffices to show that this is impossible.

Assume that $\mu$ is ergodic, let $P$ denote the distribution generated by $\mu$, and choose $\alpha \in \Sigma_{P} \cap(1 / \log a) \mathbb{Z}$, which is possible by Theorem 3.13. Then by Proposition 2.9, $\theta_{\alpha}(f \mu)$ is singular with respect to Lebesgue measure, while by Theorem 2.7, $\theta_{\alpha}(v)$ is absolutely continuous. Thus the two are not equivalent. Note that for this argument we did not require ergodicity of $v$.

Assume instead that $f$ is affine (but $\mu, v$ need not be ergodic). We first disintegrate $\mu$ according to the partition of $[0,1]$ determined by the level sets of $x \mapsto P_{x}$, where $P_{x}$ is the distribution generated by $\mu$ at $x$. Since $P_{x}$ depends only on $\mu^{(x)}$, this is a coarsening of the ergodic decomposition. Decompose $v$ similarly. By Lemma 2.3, $f$ respects these partitions, so it suffices to prove the result for the corresponding conditional measures, which in the case of $\mu$ are $T_{a}$-invariant, and $T_{b}$-invariant in the case of $v$. Hence we may assume from the start that $\mu, v$ generate a single distribution $P$ a.e. The result now follows as above from the second part of Proposition 2.9.

The case of $C^{1}$-maps when $\mu, v$ satisfy some spectral assumptions was sketched after Theorem 2.5.

Proof of Corollary 1.5. Suppose $\varphi f \varphi^{-1}=T_{a}$ and $\psi g \psi^{-1}=T_{b}$ for $\varphi, \psi \in \operatorname{diff}^{2}(\mathbb{R} / \mathbb{Z})$. Let $\mu$ be a common ergodic measure of positive dimension. The measures $\varphi \mu, \psi \mu$ are invariant, respectively, for $T_{a}$ and $T_{b}$, and the dimension hypothesis implies that they have no ergodic component of entropy zero. Now $\psi \varphi^{-1}(\varphi \mu)=\psi \mu$ and $\psi \varphi^{-1} \in \operatorname{diff}^{2}(\mathbb{R} / \mathbb{Z})$, so Theorem 1.4 implies that $\varphi \mu$ is Lebesgue. Therefore $\psi \varphi^{-1}(\varphi \mu)$ is a $T_{b}$-invariant measure equivalent to Lebesgue, so it must be Lebesgue measure as well, and so $\psi \varphi^{-1}$ preserves Lebesgue measure. The only diffeomorphism on $\mathbb{R} / \mathbb{Z}$ with this property is a rotation. 


\section{Construction and properties of the scenery flow}

Throughout this section we fix an integer $b>1$ and a non-atomic probability measure $\mu$ on $[0,1]$ which is invariant under $T_{b}$. We write $[u ; v)=[u, v) \cap \mathbb{Z}$, and similarly $[u ; v]$ etc. Our convention is $\mathbb{N}=\{1,2,3 \ldots\}$.

\subsection{The extended scenery flow}

For the moment fix the dimension $d=1$ and consider measures on $\mathbb{R}$. We use $*$ to denote the operation of normalizing a measure on $[-1,1]^{d}$, that is, if $\tau$ is a Radon measure on $\mathbb{R}^{d}$ and $\tau([-1,1])>0$, then

$$
\tau^{*}=\frac{1}{\tau([-1,1])} \tau .
$$

Thus $\tau^{\square}=\left.\tau^{*}\right|_{[-1,1] \text {. Let }} \mathcal{M}^{*} \subseteq \mathcal{M}$ denote the set of measures giving unit mass to $[-1,1]$. Write $S_{t}^{*}: \mathcal{M}^{*} \rightarrow \mathcal{M}^{*}$ for the partially defined map

$$
S_{t}^{*} \mu=\left(S_{t} \mu\right)^{*} \text {. }
$$

Thus $S^{*}=\left(S_{t}^{*}\right)_{t \in \mathbb{R}}$ is a measurable flow on the Borel subset of measures $\mu \in \mathcal{M}^{*}$ with 0 in their support.

While working with $S^{*}$ is more natural than with $S^{\square}$, we used the latter in the definition of the scenery flow because $\mathcal{P}\left(\mathcal{M}^{*}\right)$ does not carry a nice topology with which to define equidistribution of $S^{*}$-orbits. However there is a simple way to move between invariant distributions of the two flows. First, one may verify that $\tau \mapsto \tau^{\square}$ is a factor map from the measurable flow $\left(\mathcal{M}^{*}, S^{*}\right)$ to the semi-flow $\left(\mathcal{M}^{\square}, S^{\square}\right)$, i.e. $S_{t}^{\square}\left(\mu^{\square}\right)=\left(S_{t}^{*} \mu\right)^{\square}$, and so an $S^{*}$-invariant distribution $Q$ is pushed via $\mu \mapsto \mu^{\square}$ to an $S^{\square}$-invariant distribution $P=Q^{\square}$ called the restricted version of $Q$. Conversely, if $P$ is a an $S^{\square}$-invariant distribution then there is a unique $S^{*}$-invariant distribution $Q$ on $\mathcal{M}^{*}$, called the extended version of $P$, satisfying $Q^{\square}=P$. The extended version may be obtained as the inverse limit of the diagram

$$
\cdots \stackrel{s_{1}^{\square}}{\longrightarrow} \mathcal{M}^{\square} \stackrel{s_{1}^{\square}}{\longrightarrow} \mathcal{M}^{\square} \stackrel{s_{1}^{\square}}{\longrightarrow} \mathcal{M}^{\square}
$$

(so dynamically $Q$ is the natural extension of $P$ ). Indeed, starting from a left-infinite sequence $\left(\ldots, \mu_{-2}, \mu_{-1}, \mu_{0}\right)$ with $S_{1}^{\square} \mu_{i+1}=\mu_{i}$, there is a unique measure $\mu_{\infty} \in \mathcal{M}^{*}$ such that $\left.\mu_{\infty}\right|_{\left[-b^{n}, b^{n}\right]}=S_{n}^{*} \mu_{-n}$, and the induced distribution $Q$ on these measures is seen to be $S^{*}$-invariant and satisfy $Q^{\square}=P$.

We shall usually not make the distinction between the extended and restricted versions of these flows. We note for later use that they have the same pure point spectrum.

\subsection{Construction of the discrete scenery flow}

In this section we construct a flow associated to a $T_{b}$-invariant measure, and study its properties. Let $\Omega=\Omega_{b}=\{0, \ldots, b-1\}^{\mathbb{Z}}$, and denote the shift map on $\Omega$ by $T$, i.e. $(T \omega)_{i}=\omega_{i+1}$. We write $\omega_{I}$ for the subsequence $\left(\omega_{i}\right)_{i \in I}$. 
Let $\xi_{k}: \Omega \rightarrow\left[0, b^{-k}\right]$ denote the map

$$
\xi_{k}(\omega)=\sum_{i=k+1}^{\infty} b^{-i} \omega_{i}
$$

In particular we write

$$
\xi=\xi_{0},
$$

which is the base- $b$ coding map taking $\omega \in \Omega$ to the point $x \in[0,1]$ whose base- $b$ expansion is $0 . \omega_{1} \omega_{2} \ldots$ (note that this map is everywhere uncountable-to-one since it discards the non-positive coordinates of $\omega$ ).

Every non-atomic $T_{b}$-invariant measure $\mu$ lifts to a unique $T$-invariant measure $\tilde{\mu}$ on $\Omega$ such that $\xi \tilde{\mu}=\mu$; the system $(\tilde{\mu}, T)$ is a realization of the natural extension of $\left(\mu, T_{b}\right)$. For $x \in[0,1]$ we denote by $\mu^{(x)}$ the ergodic component of $x$ in $\mu$, and write $\tilde{\mu}^{(x)}$ for the unique ergodic component of $\tilde{\mu}$ which maps under $\xi_{0}$ to $\mu^{(x)}$. We also write $\widetilde{\mu}^{(\omega)}$ for the ergodic component of $\omega \in \Omega$.

We next construct a map $\pi: \Omega \rightarrow \mathcal{M}, \omega \mapsto \mu_{\omega}$, which is defined a.e. for every $T$ invariant measure on $\Omega$. Let $\mu$ be a $T_{b}$-invariant measure. Given a left-infinite sequence $\omega_{(-\infty ; k]} \in\{0, \ldots, b-1\}^{(-\infty ; k]}$ let $\tilde{\mu}\left(\cdot \mid \omega_{(-\infty ; k]}\right)$ be the probability measure obtained by conditioning $\tilde{\mu}$ on the set $\left\{\eta \in \Omega: \eta_{(-\infty ; k]}=\omega_{(-\infty ; k]}\right\}$. Note that this set can be identified, using $\xi_{k}$, with $\left[0, b^{-k}\right]$. Define the measure $\mu\left(\cdot \mid \omega_{(-\infty ; k]}\right)$ on $\left[0, b^{-k}\right]$ by pushing these conditional measures forward through $\xi_{k}$, i.e.

$$
\mu\left(\cdot \mid \omega_{(-\infty ; k]}\right)=\tilde{\mu}\left(\xi_{k}^{-1}(\cdot) \mid \omega_{(-\infty ; k]}\right) .
$$

Note that this definition depends only on the ergodic component $\tilde{\mu}^{(\omega)}$, rather than the pair $(\tilde{\mu}, \omega)$.

For any measurable $A \subseteq\left[0, b^{-k}\right]$ we have the relation

$$
\mu\left(A \mid \omega_{(-\infty ; k]}\right)=c_{\omega}^{k} \cdot \mu\left(A+b^{-k} \omega_{k} \mid \omega_{(-\infty ; k-1]}\right)
$$

where $c_{\omega}^{k}=1 / \mu\left(\omega_{k} \mid \omega_{k-1}^{\infty}\right)$ (this constant is chosen so that equality holds for $A=$ $\left.\left[0, b^{-k}\right]\right)$. More generally, for $k<m$ and $A \subseteq\left[0, b^{-m+1}\right]$, we have

$$
\mu\left(A \mid \omega_{(-\infty ; m]}\right)=c_{\omega}^{k, m} \cdot \mu\left(A+\sum_{k<i \leq m} b^{-i} \omega_{i} \mid \omega_{(-\infty ; k]}\right)
$$

where $c_{\omega}^{k, m}=c_{\omega}^{k+1} \cdot \ldots \cdot c_{\omega}^{m}$.

It follows that the sequence of measures $\mu_{\omega, k} \in \mathcal{M}$ defined for $k \leq 0$ by

$$
\mu_{\omega, k}(A)=c_{\omega}^{k, 0} \cdot \mu\left(A+\sum_{i=k+1}^{\infty} b^{-i} \omega_{i} \mid \omega_{(-\infty, k]}\right)
$$

agree as $k \rightarrow-\infty$ on the increasing sequence of intervals

$$
\left[-\xi_{k}(\omega), \xi_{k}(\omega)+b^{-k}\right]=\left[-\sum_{i=k+1}^{\infty} b^{-i} \omega_{i}, \sum_{i=k+1}^{\infty} b^{-i} \omega_{i}+b^{-k}\right]
$$


and vanish outside of them. Therefore, as $k \rightarrow-\infty$ the measures $\mu_{\omega, k}$ converge to a Radon measure which we denote

$$
\mu_{\omega}=\lim _{k \rightarrow-\infty} \mu_{\omega, k}
$$

Recall that a measure on $\mathbb{R}$ has exact dimension $\alpha$ if its local dimension exists a.e. and is a.e. equal to $\alpha$.

Theorem 3.1. For every $T_{b}$-invariant measure $\mu$, the following hold:

(i) $\mu^{(x)}$ can be represented as

$$
\mu^{(x)}=\left.\int\left(U_{-\xi(\omega)} \mu_{\omega}\right)\right|_{[0,1]^{d}} d \tilde{\mu}^{(x)}(\omega)
$$

and in particular

$$
\mu=\left.\int\left(U_{-\xi(\omega)} \mu_{\omega}\right)\right|_{[0,1]^{d}} d \tilde{\mu}(\omega) .
$$

(ii) The map $\pi^{*}: \omega \mapsto \mu_{\omega}^{*}$ intertwines the actions of $T$ and $S_{\log b}^{*}$, i.e.

$$
S_{\log b}^{*} \mu_{\omega}^{*}=\mu_{T \omega}^{*} .
$$

In particular, the distribution

$$
\widetilde{P}_{x}=\pi^{*} \tilde{\mu}^{(x)}
$$

is $S_{\log b}^{*}$-invariant, and the map

$$
\pi^{*}:\left(\Omega, \widetilde{\mu}^{(x)}, T\right) \rightarrow\left(\mathcal{M}^{*}, \widetilde{P}_{x}, S_{\log b}^{*}\right)
$$

is a factor map of discrete-time systems.

(iii) $\mu_{\omega}$ has exact dimension $h\left(\tilde{\mu}^{(\omega)}\right) / \log b$.

Proof. From equation (2) we see that for $k<0$,

$$
\mu_{\omega, k}\left(A-\xi_{0}(\omega)\right)=c_{\omega}^{k, 0} \cdot \mu\left(A+\sum_{i=k+1}^{0} b^{-i} \omega_{i} \mid \omega_{(-\infty, k]}\right)
$$

which, from equation (1), implies

$$
\mu_{\omega}\left(A-\xi_{0}(\omega)\right)=\mu\left(A \mid \omega_{(-\infty, 0]}\right) .
$$

Integrating over $\omega \sim \tilde{\mu}^{(x)}$ or over $\omega \sim \tilde{\mu}$ gives (i).

Note that $0 \in \operatorname{supp} \mu_{\omega}$ and (i) implies

$$
\mu_{T \omega}(A)=c_{\omega} \mu_{\omega}\left(\frac{1}{b} A\right)
$$

for some constant $c_{\omega}$ independent of $A$. Thus by (4), the map $\omega \mapsto \mu_{\omega}^{*}$ is defined $\tilde{\mu}$-a.e. and intertwines the shift $T$ and the scaling map $S_{\log b}^{*}$, i.e.

$$
S_{\log b}^{*} \mu_{\omega}^{*}=\mu_{T \omega}^{*} .
$$

This establishes (ii) (the later statements in that part follow from the first). 
Let

$$
\left[\omega_{1} \ldots \omega_{n}\right]=\left\{\eta \in \Omega: \eta_{1} \ldots \eta_{n}=\omega_{1} \ldots \omega_{n}\right\}
$$

denote the cylinder set corresponding to a finite sequence $\omega_{1} \ldots \omega_{n}$. A variant of the Shannon-McMillan-Breiman theorem (actually, a direct application of the ergodic theorem and the definition of entropy) states that for $\tilde{\mu}$-a.e. $\omega$,

$$
\lim _{n \rightarrow \infty} \frac{1}{n} \log \widetilde{\mu}\left(\left[\omega_{1}, \ldots, \omega_{n}\right] \mid \omega_{(-\infty ; 0]}\right)=h\left(\widetilde{\mu}^{(\omega)}\right) .
$$

As a consequence for $\tilde{\mu}$-a.e. $\omega$ the measure $\tau=\mu\left(\cdot \mid \omega_{(-\infty, 0]}\right)$ has exact dimension $h\left(\widetilde{\mu}^{(\omega)}\right) / \log b$, i.e.

$$
\lim _{r \searrow 0} \frac{\log \tau\left(B_{r}(x)\right)}{\log r}=\frac{h\left(\tilde{\mu}^{(\omega)}\right)}{\log b} \quad \text { at } \tau \text {-a.e. point } x
$$

(what is obvious is that this limit holds when, instead of $B_{r}(x)$, we consider the mass of $b$-adic intervals containing $x$, since these correspond to cylinder sets; the version above follows using e.g. [17, Theorem 15.3]). The same argument also holds for $\mu\left(\cdot \mid \omega_{(-\infty, k]}\right)$ and for any $k<0$, hence for $\mu_{\omega, k}$, and gives the result for $\mu_{\omega}$. This proves (iii).

As a special case of the last part of the theorem, we remark that when $h(\tilde{\mu})=0$ all the conditional measures $\mu\left(\cdot \mid \omega_{(-\infty, k]}\right)$ consist of a single atom, and consequently $\mu_{\omega}=\delta_{0}$. Likewise, when $\tilde{\mu}$ is $\lambda^{*}$ it is easy to verify that $\mu_{\omega}=\lambda^{*}$ for $\tilde{\mu}$-a.e. $\omega$. In these cases the flows $P_{x}$ are trivial, consisting of point masses at the $S^{*}$-fixed points $\delta_{0}$ or $\lambda^{*}$.

\subsection{Convergence of the scenery to the scenery flow}

We now turn to the sceneries of $T_{b}$-invariant measures and their relation to the flow constructed above. We continue to work in dimension $d=1$ and with the notation of the previous section. In particular $\mu$ is a $T_{b}$-invariant measure and $\widetilde{\mu}, \widetilde{P}_{x}$ are as in Theorem 3.1. For $\mu$-typical $x$ let

$$
P_{x}=\int_{0}^{1} S_{t \log b}^{*} \widetilde{P}_{x} d t
$$

Note that by Proposition 2.4, $\widetilde{P}_{x}$ is $S_{\log b}^{*}$-invariant and ergodic.

Let us introduce another sequence of measures $\mu_{\omega, k}^{\prime}$ on $\left[0, b^{-k}\right]$ by

$$
\mu_{\omega, k}^{\prime}(A)=c_{\omega}^{k, 0} \cdot \tilde{\mu}\left(\xi_{k}^{-1}\left(A+\sum_{i=k+1}^{\infty} b^{-i} \omega_{i}\right)\right)
$$

This is the same as the definition of $\mu_{\omega, k}$ except we have not conditioned on $\omega_{(-\infty, k]}$.

Proposition 3.2. $\lim _{k \rightarrow-\infty} \mu_{\omega, k}^{\prime}=\mu_{\omega}$ weak-* on any compact set in $\mathbb{R}$. 
Proof. Let $\tilde{\mu}\left(\cdot \mid \omega_{[k+1 ; \infty)}\right)$ denote the conditional measure $\tilde{\mu}$ on sequences $\eta \in \Omega$ given the "future" $\eta_{[k+1 ; \infty)}=\omega_{[k+1 ; \infty)}$. Then

$$
\begin{aligned}
\mu_{\omega, k}^{\prime} & =\int \mu_{\eta, k} d \widetilde{\mu}\left(\eta \mid \omega_{[k+1 ; \infty)}\right) \\
& =\int\left(\mu_{\eta, k}-\mu_{\eta}\right) d \widetilde{\mu}\left(\eta \mid \omega_{[k+1 ; \infty)}\right)+\int \mu_{\eta} d \widetilde{\mu}\left(\eta \mid \omega_{[k+1 ; \infty)}\right)
\end{aligned}
$$

The second term in the last expression is a measure-valued martingale in the variable $\omega$ with respect to the filtration $\mathcal{F}_{k} \subseteq \mathcal{F}_{k-1} \subseteq \cdots$, where $\mathcal{F}_{k}$ is the $\sigma$-algebra generated by coordinates $k+1, k+2, \ldots$ Since these algebras generate the Borel algebra on $\Omega$, the term on the right converges $\tilde{\mu}$-a.e. to $\mu_{\omega}$ as $k \rightarrow-\infty$.

In order to deal with the first term on the right, note that if we integrate against any compactly supported function $f$ on $\mathbb{R}$, for $k>k_{0}(\omega)$ the measures $\mu_{\eta, k}$ and $\mu_{\eta,-\infty}$ will agree on the support of $f$, and so the integral will vanish. Hence the first term also converges to 0 weak-* on any compact set.

Corollary 3.3. $\lim _{k \rightarrow-\infty}\left(\mu_{\omega, k}^{\prime}\right)^{\square}=\left(\mu_{\omega}\right)^{\square}$ in the weak-* sense for $\tilde{\mu}$-a.e. $\omega$.

For the next step we rely on a classical ergodic theorem due to Maker:

Theorem 3.4 (Maker, [15]). Let $(\Omega, v, T)$ be a measure preserving system. Let $F_{n}$ be measurable functions with $\sup _{n}\left|F_{n}\right| \in L^{1}$ and suppose that $F_{n} \rightarrow F$ a.e. Then

$$
\frac{1}{N} \sum_{n=1}^{N} T^{n} F_{n} \rightarrow \mathbb{E}(F \mid \mathcal{E})
$$

a.e., where $\mathcal{E}$ is the $\sigma$-algebra of $T$-invariant sets.

Proposition 3.5. For $\mu$-a.e. $x$,

$$
\lim _{N \rightarrow \infty} \frac{1}{N} \sum_{n=1}^{N} \delta_{\mu_{x, n} \log b} \rightarrow \widetilde{P}_{x}
$$

Proof. Define $F_{k}(\omega)=\delta_{\left(\mu_{\omega,-k}^{\prime}\right)} \square$ and $F_{k}(\omega)=\delta_{\left(\mu_{\omega}\right)} \square$. From the corollary we know that $F_{k} \rightarrow F$ a.s. and these distribution-valued functions are uniformly bounded in the space of distributions on $\mathcal{P}\left(\mathcal{M}^{\square}\right)$. Thus by Maker's theorem,

$$
\frac{1}{N} \sum_{n=1}^{N} F_{k}\left(T^{k} \omega\right) \rightarrow \mathbb{E}(F \mid \mathcal{E})
$$

$\tilde{\mu}$-a.e. One verifies from the definitions that

$$
\left(\mu_{T^{k} \omega, k}^{\prime}\right)^{\square}=\mu_{x, k \log k}
$$

for $x=\xi_{0}(\omega)$. The proposition follows, since $\mathbb{E}\left(\delta_{\left(\mu_{\omega}\right)} \square \mid \mathcal{E}\right)=\widetilde{P}_{x}$. 
Proposition 3.6. $\mu$ generates $P_{x}$ at $\mu$-a.e. $x$. In particular, the scenery flow generated by $\mu$ at $x$ depends only on the ergodic component of $x$ and arises as a factor of the $\log b$-suspension of $\left(\Omega, \tilde{\mu}^{(x)}, T\right)$.

Proof. The first statement follows by applying the operator $\int_{0}^{1} S_{t \log b}^{\square} d t$ to the limit in the proposition above. For the second statement, note that from the definition of $P_{x}$ it follows immediately that $P_{x}$ is $S^{*}$-invariant and ergodic, and that $\left(\mathcal{M}^{*}, P_{x}, S^{*}\right)$ is a factor of the $\log b$-suspension of the discrete time system $\left(\mathcal{M}^{*}, \widetilde{P}_{x}, S_{\log b}^{*}\right)$. Since the latter is a factor of $\left(\Omega, \widetilde{\mu}^{(x)}, T\right)$ by Theorem 3.1 , the proof is complete.

\subsection{The multidimensional case}

We now turn to the higher-dimensional setting. Let $*$ denote the normalization operation $\mu \mapsto \mu^{*}=\frac{1}{\mu\left([-1,1]^{d}\right)} \mu$ and define the associated objects as in Section 3.1.

Let $\mu_{1}, \ldots, \mu_{d}$ be $T_{b}$-invariant measures and write $\mu=\mu_{1} \times \cdots \times \mu_{d}$, which is a measure on $[0,1]^{d}$ invariant under the diagonal map $T_{b}(x)=\left(T_{b} x_{1}, \ldots, T_{b} x_{d}\right)$. The measure $\tilde{\mu}=\tilde{\mu}_{1} \times \cdots \times \tilde{\mu}_{d}$ on $\Omega^{d} \cong\left(\{0, \ldots, b-1\}^{d}\right)^{\mathbb{Z}}$ is the natural extension of $\mu$. We may define maps $\xi_{k}: \Omega^{d} \rightarrow\left[0, b^{-k}\right]^{d}$ by applying $\xi_{k}$ coordinatewise and define a map $\omega \mapsto \mu_{\omega} \in \mathcal{M}_{d}$ using the same procedure as in dimension $d=1$.

Let $\varphi_{i}$ denote projection to the $i$-th coordinate. Let $\pi^{*}$ and $\pi_{i}^{*}$ denote the maps $\omega \mapsto \mu_{\omega}^{*}$ and $\eta \mapsto\left(\mu_{i}\right)_{\eta}^{*}$ respectively.

Proposition 3.7. Let $\mu_{1}, \ldots, \mu_{d}$ be $T_{b}$-invariant measures on $[0,1]$, let $\mu=\times_{i=1}^{d} \mu_{i}$, and let $\omega \mapsto \mu_{\omega}$ be as above. Then:

(i) The analogs of Theorem 3.1 and Proposition 3.6 hold.

(ii) $\mu_{\omega}=\times_{i=1}^{d}\left(\mu_{i}\right)_{\omega^{i}}$.

(iii) If $\mu$ generates $\widetilde{P}_{x}$ at $x \in[0,1]^{d}$ and $\mu_{i}$ generates $\widetilde{P}_{i, y}$ at $y$, and we identify product measures with $d$-tuples of measures, then the following diagram of factor maps commutes:

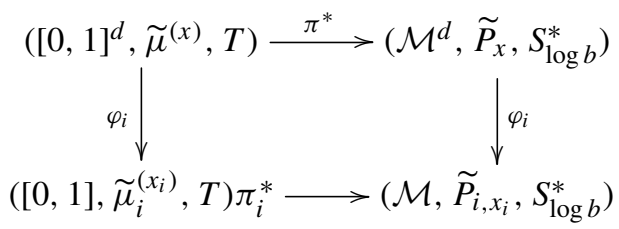

Proof. The proof of (i) is the same as in the 1-dimensional case.

For (ii), note that the space of product measures on $\mathbb{R}^{d}$ is closed and each $\mu_{x, t}$ is a product measure, so the scenery flow of a product measure is supported on product measures. Hence by (i), $\mu_{\omega}$ is a product measure.

In order to see that $\mu_{\omega}=\times_{i=1}^{d} \mu_{i, \omega^{i}}$, let $Q$ be the $S_{\log b}^{*}$-invariant distribution on pairs of measures obtained by pushing forward $\tilde{\mu}$ through the map $\omega \mapsto\left(\left(\mu_{1}\right)_{\omega^{1}}^{*}, \mu_{\omega}^{*}\right)$, and let $Q_{x}$ be the push-forward of $\tilde{\mu}^{(x)}$ by the same map, so that $Q=\int Q_{x} d \tilde{\mu}(x)$. We wish to 
show that $Q$-a.e. $\operatorname{pair}\left(\tau, v_{1} \times \cdots \times v_{d}\right)$ satisfies $\tau=v_{1}$, so we must show that for $\mu$-a.e. $x$ this holds for $Q_{x}$. To see this, consider for $\mu$-typical $x \in[0,1]^{d}$ the sequence

$$
\left(\tau_{n}, v_{n}\right)=\left(\left(\mu_{1}\right)_{x_{1}, n \log b}, \mu_{x, n \log b}\right), \quad n=1,2, \ldots,
$$

and repeat the proof of Proposition 3.6 to conclude that $\frac{1}{N} \sum_{n=1}^{N} \delta_{\left(\tau_{n}, v_{n}\right)} \rightarrow Q_{x}$. Since the relationship $\tau_{n}=\pi_{1}\left(v_{n}\right)$ holds for all $n$ and this is a closed condition, it also holds for the limiting distribution $Q_{x}$.

Finally, the commutativity of the diagram is a direct result of the relationship $\left(\mu_{i}\right)_{\omega^{i}}$ $=\pi_{i}\left(\mu_{\omega}\right)$.

\subsection{Eigenvalues and ergodicity of flows}

We briefly present some technical facts about flows and their spectrum and ergodicity properties. For the sake of economy we present the discussion for an invariant distribution $P$ on $\left(\mathcal{M}^{*}, S^{*}\right)$.

A function $f \in L^{2}(P)$ is generally defined only $P$-a.e. and hence for typical $v$ it is defined at $S_{t}^{*} v$ for only Lebesgue-a.e. $t$. The next lemma says that a function which behaves like an eigenfunction at a.e. point along a.e. orbit may be modified on a set of measure zero to become an eigenfunction.

Lemma 3.8. Let $\varphi \in L^{2}(P)$ and suppose that for every $t \in \mathbb{R}$ we have $S_{t}^{*} \varphi=e(\alpha t) \varphi$ $P$-a.e. Then there exists $\bar{\varphi} \in L^{2}(P)$ which, for $P$-a.e. $v$, satisfies $S_{t}^{*} \bar{\varphi}(\nu)=e(\alpha t) \varphi(v)$ for every $t \in \mathbb{R}$, and $\varphi=\bar{\varphi}$ a.e.

Proof. Define $\bar{\varphi}(\nu)=\int_{0}^{1} e(-\alpha t) \varphi\left(S_{t}^{*} v\right) d t$.

Usually, the ergodic decomposition of a measure is defined only in an a.e. sense. For the decomposition of $P$ with respect to $S_{t_{0}}^{*}$ we can give a more canonical description. We say that an $S_{t_{0}}^{*}$-invariant distribution $Q$ is an ergodic component of $P$ (with respect to $S_{t_{0}}^{*}$ ) if it is ergodic for $S_{t_{0}}^{*}$ and $\int_{0}^{1} S_{t_{0} \cdot t}^{*} Q d t=P$. Note that if $P=\int Q_{\nu} d P(\nu)$ is an abstract ergodic decomposition of $P$ with respect to $S_{t_{0}}^{*}$ then $P=\int\left(\int_{0}^{1} S_{t_{0} \cdot t}^{*} Q_{\nu} d t\right) d P(v)$, and each of the inner integrals is $S^{*}$-invariant. Therefore, ergodicity of $P$ implies that for $P$-a.e. $v$ the inner integral is $P$, so $Q_{v}$ is an ergodic component. Hence ergodic components exist.

Lemma 3.9. If $Q$ and $Q^{\prime}$ are ergodic components for $S_{t_{0}}^{*}$ then $S_{t_{0} \cdot r}^{*} Q^{\prime}=Q$ for some $r \in[0,1]$. In particular, the representation of $P$ as $\int_{0}^{1} S_{t_{0} \cdot t}^{*} Q d t$ does not depend (up to a translation modulo 1 of the parameter space) on the ergodic component $Q$.

Proof. Since $\int_{0}^{1} S_{t_{0} \cdot t}^{*} Q d t$ and $\int_{0}^{1} S_{t_{0} \cdot t}^{*} Q^{\prime} d t$ are both ergodic decompositions of $P$, by uniqueness of the ergodic decomposition we see that for a.e. $t \in[0,1]$ there is an $s \in$ $[0,1]$ with $S_{t_{0} \cdot t}^{*} Q=S_{t_{0} \cdot s}^{*} Q^{\prime}$. Then for $r=s-t$ (or $r=1+s-t$ if $s<t$ ), we have $Q=S_{t_{0}(s-t)}^{*} Q^{\prime}$. The second statement is immediate from the first.

Lemma 3.10. Let $Q$ be an ergodic component of $P$ with respect to $S_{t_{0}}^{*}$. Then either $Q=P$, i.e. $S_{t_{0}}^{*}$ is ergodic, or there is a largest $n \in \mathbb{N}$ such that $Q$ is invariant under $S_{t_{0} / n}^{*}$. 
Proof. Consider the map $q: \mathbb{R} / \mathbb{Z} \rightarrow \mathcal{P}(\mathcal{M})$ given by $q(t)=S_{t_{0} \cdot t}^{*} Q$, which is well defined since $S_{t_{0}}^{*} Q=Q$. Let $\Lambda \subseteq \mathbb{R} / \mathbb{Z}$ denote the set of periods of $q$, that is, $r \in \Lambda$ if $q(t+r)=q(t)$ for $t \in \mathbb{R} / \mathbb{Z}$, or equivalently, $q(r)=q(0)$. Since $q$ is measurable with respect to Lebesgue measure, either $\Lambda$ is discrete or $\Lambda=\mathbb{R} / \mathbb{Z}$. In the latter case, $P=\int_{\mathbb{R} / \mathbb{Z}} q(t) d t=q(0)=Q$. In the former case $\Lambda$ has the form $\{k / n: 0 \leq k<n\}$ for some $n$, and this is the $n$ we are looking for.

Lemma 3.11. For $t_{0}>0$ the following are equivalent:

(i) $1 / t_{0} \in \Sigma_{P}$.

(ii) For some (equivalently every) ergodic component $Q$ of $S_{t_{0}}^{*}$, the flow $\left(\mathcal{M}^{*}, P, S^{*}\right)$ is isomorphic to the $t_{0}$-suspension of $\left(\mathcal{M}^{*}, Q, S_{t_{0}}^{*}\right)$.

(iii) $S_{t_{0}}^{*}$ is not ergodic, and its ergodic components are not preserved under $S_{t_{0} / n}^{*}$ for any $n \in \mathbb{N}$.

Proof (sketch). (i) $\Rightarrow$ (ii): If $\varphi$ is an eigenfunction for $1 / t_{0}$ then one may verify that the ergodic components of $S_{t_{0}}^{*}$ are precisely the conditional distributions of $P$ on the level sets of $\varphi$. Fixing an ergodic component $Q$ supported on a level set $\varphi^{-1}(e(s))$ let $r: \mathcal{M}^{*} \rightarrow$ $\left[0, t_{0}\right)$ be the $P$-a.e. defined function such that $e\left(r(v) / t_{0}\right)=\varphi(v) / e(s)$, so for $Q$-a.e. $v$ and $0 \leq t<t_{0}$ we have $r\left(S_{t}^{*} v\right)=t$. Then $v \mapsto\left(S_{-r(v)}^{*} v, \varphi(v)\right)$ is an isomorphism of $\left(\mathcal{M}^{*}, P, S^{*}\right)$ and the $t_{0}$-suspension of $\left(\mathcal{M}^{*}, Q, S_{t_{0}}^{*}\right)$.

(ii) $\Rightarrow$ (iii): Trivial since e.g. the subset of $\mathcal{M}^{*}$ corresponding to $\mathcal{M}^{*} \times\left[0, t_{0} / 2\right)$ in the suspension is $S_{t_{0}}^{*}$-invariant, but not $S_{t_{0} / n}^{*}$-invariant for any $1 \neq n \in \mathbb{N}$.

(iii) $\Rightarrow$ (i): By the previous lemma we find that the action of $S^{*}$ on the ergodic components for $S_{t_{0}}^{*}$ is isomorphic to $\left[0, t_{0}\right)$ with addition modulo $t_{0}$. As $P$-a.e. point belongs to a well defined ergodic component, this gives an eigenfunction with eigenvalue $1 / t_{0}$.

From part (ii) and the proof of the implication (i) $\Rightarrow($ ii), we have:

Corollary 3.12. Let $1 / t_{0} \in \Sigma_{P}$ with associated eigenfunction $\varphi$, and suppose that $Q, Q^{\prime}$ are ergodic components for $S_{t_{0}}^{*}$. Then $\varphi$ is almost surely constant for each of the distributions $Q, Q^{\prime}$, with a.s. value denoted $\varphi(Q), \varphi\left(Q^{\prime}\right)$, respectively, and $Q=S_{t_{0} \cdot r}^{*} Q^{\prime}$, where $r \in[0,1]$ is such that $e^{2 \pi i r}=\varphi(Q) / \varphi\left(Q^{\prime}\right)$.

\subsection{The spectrum of $\left(\mathcal{M}^{*}, P_{x}, S^{*}\right)$}

In this section we prove Theorem 2.5 . Let $\mu$ be $T_{b}$-ergodic with entropy strictly between 0 and $\log b$. Recall the construction and notation from Section 3.2: specifically $(\Omega, \tilde{\mu}, T)$ is the natural extension of $\left([0,1], \mu, T_{b}\right)$, the image of $\tilde{\mu}$ under $\omega \mapsto \mu_{\omega}$ is the $S_{\log b}^{*}$-ergodic distribution $\widetilde{P}$ (it does not depend on $x$ or $\omega$, as it did in previous sections, because $\mu$ and $\tilde{\mu}$ are now ergodic), and $P=\int_{0}^{1} S_{t \log b}^{*} \widetilde{P} d t$ is the distribution of the scenery flow of $\mu$.

Recall that the lower Hausdorff dimension of a measure $\tau$ is

$$
\underline{\operatorname{dim}} \tau=\inf \{\operatorname{dim} A: \tau(A)>0\} .
$$

We note that if $\tau$ has exact dimension $\alpha$ then $\underline{\operatorname{dim}} \tau=\alpha$ as well. 
It is simple to verify that $\tau \ll \tau^{\prime}$ implies $\underline{\operatorname{dim}} \tau \geq \underline{\operatorname{dim}} \tau^{\prime}$ and, more generally, if a measure $\tau$ can be written as $\tau=\int \tau_{i} d \sigma(i)$, then $\underline{\operatorname{dim}} \tau \geq \operatorname{essinf}_{i \sim \sigma} \underline{\operatorname{dim}} \tau_{i}$. Thus if $f$ is a map then $f \tau=\int f \tau_{i} d \sigma(i)$, and a similar bound applies.

We are out to show that

$$
\frac{n}{\log a} \mathbb{Z} \subseteq \Sigma_{P_{x}} \subseteq \frac{1}{\log a} \Sigma_{\mu(x)} \cup \frac{n}{\log a} \mathbb{Z} .
$$

The right hand inclusion follows from the fact that $\left(\mathcal{M}^{*}, P_{x}, S^{*}\right)$ is a factor of the $\log b$ suspension of $\left([0,1], \mu, T_{b}\right)$. To establish the left hand inclusion it suffices to prove the following theorem.

Theorem 3.13. There exists an $n \in \mathbb{N}$ such that $n / \log a \in \Sigma_{\left(\mathcal{M}^{*}, P, S^{*}\right)}$.

Proof. Since $\widetilde{P}$ is an ergodic component of $P$ with respect to $S_{\log a}^{*}$, by Lemmas 3.10 and 3.11 it suffices to show that $\widetilde{P}$ is not $S^{*}$-invariant. Suppose that it were $S^{*}$-invariant. We claim that this implies that $\mu$ is Lebesgue measure, contradicting the assumption of intermediate dimension.

To this end, choose an integer $d$ such that $d \operatorname{dim} \mu>1$ and write $\mu^{* d}$ for the $d$ fold convolution of $\mu$, which is the image of the $d$-fold product $\times_{i=1}^{d} \mu$ under the map $f(x)=\sum_{i=1}^{d} x_{i}$. We first show that $\mu^{* d}$ has dimension 1 . Recall that by Theorem 3.1,

$$
\mu=\int U_{-\xi(\omega)} \mu_{\omega} d \tilde{\mu}(\omega)
$$

Since $\widetilde{P}$ is $S^{*}$-invariant, there is a function $\xi(x, t) \in[0,1]$ such that

$$
\mu=\left.\iint_{0}^{1}\left(U_{-\xi(\omega, t)} S_{t \log b} \mu_{\omega}\right)\right|_{[0,1]} d t \tilde{\mu}(\omega) .
$$

This gives a similar representation of the product measure: write $t=\left(t_{1}, \ldots, t_{d}\right)$ and $u^{d}$ for uniform measure on $[0,1]^{d}$, and likewise write $\omega=\left(\omega^{1}, \ldots, \omega^{d}\right)$ and $\tilde{\mu}^{d}=x_{i=1}^{d} \tilde{\mu}$. Then

$$
\underset{i=1}{\stackrel{\times}{\times}} \mu=\int_{\Omega^{d}} \int_{[0,1]^{d}} \underset{i=1}{\stackrel{d}{\times}}\left(\left.\left(U_{-\xi\left(\omega^{i}, t^{i}\right)} S_{t_{i}} \log b \mu_{\omega^{i}}^{*}\right)\right|_{[0,1]}\right) d u^{d}(t) d \tilde{\mu}^{d}(\omega) .
$$

Therefore, using the comments preceding the proposition,

$$
\underline{\operatorname{dim}} \mu^{* d} \geq \underset{\omega \sim \widetilde{\mu}^{d}, t \sim u^{d}}{\operatorname{ess} \inf } f\left(\left.\underset{i=1}{\stackrel{d}{\times}}\left(U_{-\xi\left(\omega^{i}, t^{i}\right)} S_{t_{i} \log b} \mu_{\omega^{i}}^{*}\right)\right|_{[0,1]}\right) .
$$

Since $f$ is linear and $\xi\left(\omega^{i}, t^{i}\right) \in[0,1]$, the above is

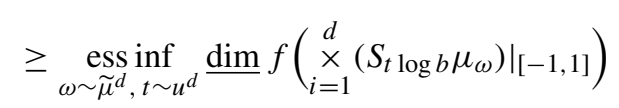


because each of the previous measures is absolutely continuous with respect to the corresponding measure above. Writing $f_{t}(x)=\sum t_{i} x_{i}$, we have, by another absolute-continuity argument,

$$
\geq \underset{\omega \sim \widetilde{\mu}^{d}}{\operatorname{essinf}}\left(\underset{t \sim u^{d}}{\operatorname{essinf}} \underline{\operatorname{dim}} f_{t}\left(\left.\left(\underset{i=1}{\stackrel{d}{\times}} \mu_{\omega^{i}}\right)\right|_{[-b, 2 b]}\right)\right) .
$$

Finally, for fixed typical $\omega$ we have

$$
\operatorname{dim} \underset{i=1}{\stackrel{d}{\times}} \mu_{\omega^{i}}=\sum_{i=1}^{d} \operatorname{dim} \mu_{\omega^{i}}>1
$$

so the inner ess inf (over $t \sim u^{d}$ ) in the previous expression is 1 by the following version of Mastrand's classical theorem on projections of measures.

Theorem 3.14 (Hunt-Kaloshin [9]). Let $\sigma$ be an exact-dimensional probability measure on $\mathbb{R}^{d}$ with $\operatorname{dim} \tau=\alpha$. Then for Lebesgue-a.e. $\left(t_{1}, \ldots, t_{d}\right) \in \mathbb{R}^{d}$, the image of $\sigma$ under $x \mapsto \sum_{i=1}^{d} t_{i} x_{i}$ is exact-dimensional and has dimension $\min \{1, \operatorname{dim} \sigma\}$.

Thus, we have shown that $\mu^{* d}$ has dimension 1 . Next, note that convolution in $\mathbb{R} / \mathbb{Z}$ is obtained by taking the convolution in $\mathbb{R}$ modulo 1 . Since this is a countable-to- 1 local isometry $\mathbb{R} \rightarrow \mathbb{R} / \mathbb{Z}$, it does not change dimension, so the $d$-th convolution of $\mu$ in $\mathbb{R} / \mathbb{Z}$ has dimension 1. Since this convolved measure is also $T_{b}$-invariant, it is exact-dimensional, and hence its exact dimension is 1 , and it must be Lebesgue measure because this is the only measure of dimension 1 invariant under $T_{b}$. Finally, by examining the Fourier coefficients and using the elementary relation $\widehat{\left(\mu^{* d}\right)}(k)=\widehat{\mu}(k)^{d}$, we conclude that $\mu$ is Lebesgue measure. This is the desired contradiction.

\subsection{The phase}

Let $\mu$ be a $T_{b}$-invariant measure of intermediate dimension generating a.e. the same (necessarily $S^{*}$-ergodic) distribution $P$. As usual we denote by $P_{x}$ the distribution generated by $\mu$ at $x$ and by $P_{x, y}$ the distribution generated by $\mu \times \mu$ at $(x, y)$, and by $\widetilde{P}_{x}$ and $\widetilde{P}_{x, y}$ the distributions obtained from $\tilde{\mu}$ and $\widetilde{\mu} \times \tilde{\mu}$ as in Theorem 3.1.

Throughout this section $\alpha \in \Sigma_{P}$ and $\varphi$ is the corresponding eigenvalue. Recall that the phase $p_{\alpha}\left(P_{x, y}\right)$ is the almost sure value of $\varphi(\sigma) / \varphi(\tau)$ for $\sigma \times \tau \sim P_{x, y}$, which is the same as for $\sigma \times \tau \sim \widetilde{P}_{x, y}$. Therefore for $\mu \times \mu$-typical $(x, y)$ and corresponding typical $(\omega, \eta) \in \Omega \times \Omega$,

$$
p_{\alpha}\left(P_{x, y}\right)=\frac{\varphi\left(\mu_{\omega}^{*}\right)}{\varphi\left(\mu_{\eta}^{*}\right)}
$$

Fixing a $\mu$-typical $x_{0}$, the phase measure $\theta_{\alpha}=\theta_{\alpha}\left(\mu, x_{0}\right)$ is the push-forward of $\mu$ via $y \mapsto p_{\alpha}\left(P_{x_{0}, y}\right)$. Thus, for $\omega_{0}$ corresponding to $x_{0}$, we find that $\theta_{\alpha}\left(\mu, x_{0}\right)$ is the pushforward of $\widetilde{\mu}$ through the map $\eta \mapsto \varphi\left(\mu_{\omega_{0}}^{*}\right) / \varphi\left(\mu_{\eta}^{*}\right)$.

Proposition 3.15. If $\lambda \in \Sigma_{P} \backslash(1 / \log b) \mathbb{Q}$ then $\theta_{\lambda}$ is Lebesgue measure. 
Proof. $\eta \mapsto \varphi\left(\mu_{\eta}^{*}\right)$ is an eigenfunction for the system $(\Omega, \tilde{\mu}, T)$ with eigenvalue $\lambda \log b$. Since $\lambda \log b$ is irrational, the distribution of $\varphi\left(\mu_{\eta}^{*}\right)$ is uniform on the circle, so the same is true for $\varphi\left(\mu_{\omega_{0}}^{*}\right) / \varphi\left(\mu_{\eta}^{*}\right)$, and the conclusion follows.

Proposition 3.16. If $\alpha=n /(m \log b) \in \Sigma_{\left(\mathcal{M}^{*}, P, S^{*}\right)}$ and $\mu$ is ergodic then $\theta_{\alpha}$ is uniform measure on a rotation of the $m$-th roots of unity. In particular if $m=1$ then $\theta_{\alpha}$ consists of a single atom.

Proof. $\eta \mapsto \varphi\left(\mu_{\eta}^{*}\right)$ is an eigenfunction of $(\Omega, \tilde{\mu}, T)$ with eigenvalue $(\log b) \alpha=n / m$. The distribution of $\varphi\left(\mu_{\eta}^{*}\right)$ for $\eta \sim \tilde{\mu}$ is just the distribution of this eigenfunction, which is uniform on a rotation of the $m$-th roots of unity. Therefore the same is true for $\varphi\left(\mu_{\omega_{0}}^{*}\right) / \varphi\left(\mu_{\eta}^{*}\right), \eta \sim \tilde{\mu}$, proving the proposition.

Now we turn to the non-ergodic case. For $z=e(t)$ with $t \in[0,1)$ let us denote $L(z)$ $=t / \alpha$.

Lemma 3.17. The distribution of $\mu_{\omega}^{*}, \omega \sim \tilde{\mu}$, is the same as the distribution of $S_{L(z)}^{*} v$, $(v, z) \sim \widetilde{P}_{x_{0}} \times \theta_{\alpha}$, where $\theta_{\alpha}=\theta_{\alpha}\left(\mu, x_{0}\right)$.

Proof. Write for brevity

$$
p(\omega)=\frac{\varphi\left(\mu_{\omega_{0}}^{*}\right)}{\varphi\left(\mu_{\omega}^{*}\right)}
$$

and consider the map $\Omega \rightarrow \mathcal{M}^{*} \times\{|z|=1\}$ defined by

$$
\omega \mapsto\left(S_{-L(p(\omega))}^{*} \mu_{\omega}^{*}, p(\omega)\right),
$$

It suffices to show that this map takes $\tilde{\mu}$ to $\widetilde{P} \times \theta_{\alpha}$, and for this we must show that (a) the second component of the image measure is $\theta_{\alpha}$, and (b) conditioned on the value of the second component, the distribution of the first component is $\widetilde{P}_{x_{0}}$.

For (a), fix $x \sim \mu$. Then for $\tilde{\mu}^{(x)}$-a.e. $\omega$ the value of $p(\omega)=\varphi\left(\mu_{\omega_{0}}^{*}\right) / \varphi\left(\mu_{\omega}^{*}\right)$ is $p_{\alpha}\left(\widetilde{P}_{x_{0}, x}\right)$, because, by definition, $\mu_{\omega_{0}}^{*} \times \mu_{\omega}^{*}$ is a typical element of $\widetilde{P}_{x, x_{0}}$; and this is the same as $p_{\alpha}\left(P_{x_{0}, x}\right)$, because $P_{x_{0}, x}=\int_{0}^{1} S_{t \log b}^{*} \widetilde{P}_{x_{0}, x} d t$. The distribution of $p_{\alpha}\left(P_{x_{0}, x}\right)$ for $x \sim \mu$ is by definition equal to $\theta_{\alpha}$.

Next, conditioned on the value $p(\omega)=\varphi\left(\mu_{\omega_{0}}^{*}\right) / \varphi\left(\mu_{\omega}^{*}\right)$ we know by Corollary 3.12 that $S_{-L(z)}^{*} \widetilde{P}_{x}=\widetilde{P}_{x_{0}}$. This proves the lemma.

Proposition 3.18. If $\alpha \in \Sigma_{P} \cap(1 / \log b) \mathbb{Q}$ then $\theta_{\alpha}$ is singular with respect to Lebesgue measure.

Proof. Our strategy is similar to the proof of Theorem 3.13. Choose an integer $d$ with $d \cdot \operatorname{dim} \mu>1$; we show that if $\theta_{\alpha}$ were absolutely continuous with respect to Lebesgue measure then this would imply that $\operatorname{dim} \mu^{* d}=1$, which would contradict the intermediate entropy of $\mu$, as in Theorem 3.13 .

We aim to show that $\operatorname{dim} \mu^{* d}=1$. By Theorem 3.1 we have

$$
\mu=\left.\int\left(U_{\xi(\omega)} \mu_{\omega}\right)\right|_{[0,1]} d \tilde{\mu}(\omega) .
$$


Write $\omega=\left(\omega^{1}, \ldots, \omega^{d}\right)$ and $\widetilde{\mu}^{d}=\times_{i=1}^{d} \tilde{\mu}$. Let $f(x)=\sum_{i=1}^{d} x_{i}$. Then

$$
\begin{aligned}
\underline{\operatorname{dim}} \mu^{* d} & =\underline{\operatorname{dim}} f\left(\left.\int \underset{i=1}{\underset{x}{x}}\left(U_{\xi\left(\omega^{i}\right)} \mu_{\omega^{i}}\right)\right|_{[0,1]} d \widetilde{\mu}^{d}(\omega)\right) \\
& \geq \underset{\omega \sim \widetilde{\mu}^{d}}{\operatorname{essinf} \operatorname{dim}} f\left(\left.\left(\underset{i=1}{\underset{x}{\times}} U_{\xi\left(\omega^{i}\right)} \mu_{\omega^{i}}\right)\right|_{[0,1]^{d}}\right) \\
& \geq \underset{\omega \sim \widetilde{\mu}^{d}}{\operatorname{ess} \inf } f\left(\left.\left(\underset{i=1}{\underset{i}{\times}} \mu_{\omega^{i}}\right)\right|_{[-1,1]^{d}}\right)
\end{aligned}
$$

where in the last equality we used linearity of $f$. Writing $v=\left(v_{1}, \ldots, v_{d}\right), z=$ $\left(z_{1}, \ldots, z_{d}\right)$ and $\widetilde{P}^{d}, \theta_{\alpha}^{d}$ for the $d$-fold product measures, we can apply the previous lemma to get

$$
=\underset{\nu \sim \widetilde{P}_{x_{0}}, t \sim \theta_{\alpha}^{d}}{\operatorname{dim}} f\left(\left.\left(\underset{i=1}{\stackrel{d}{\times}} S_{L\left(z^{i}\right)}^{*} v_{i}\right)\right|_{[-1,1]^{d}}\right) .
$$

Setting $f_{t}(x)=\sum_{i=1}^{d} e^{t_{i}} x_{i}$ and $L(z)=\left(L\left(z_{1}\right), \ldots, L\left(z_{d}\right)\right)$, we obtain

$$
\geq \underset{v \sim \widetilde{P}_{x_{0}}, t \sim \theta_{\alpha}^{d}}{\operatorname{eimf}} f_{-L(z)}\left(\left.\left(\begin{array}{cc}
d \\
x & v^{i} \\
i=1
\end{array}\right)\right|_{[-b, b]^{d}}\right) .
$$

Now with $v_{1}, \ldots, v_{d}$ fixed typical measures for $\widetilde{P}_{x_{0}}$ we know that $\times\left._{i=1}^{d} v_{i}\right|_{[-b, b]^{d}}$ has exact dimension $d \operatorname{dim} \mu>1$. Also, since $L$ is a piecewise smooth map, if $\theta_{\alpha}$ were absolutely continuous then the distribution of $L(z)$ for $z \sim \theta_{\alpha}^{d}$ would be absolutely continuous with respect to $d$-dimensional Lebesgue measure. Hence, applying Marstrand's theorem again, we find that for $\widetilde{P}_{x_{0}}^{d}$-a.e. choice of $v$ the dimension in the expression above is 1 , so the essential infimum is 1 . This completes the proof.

Acknowledgments. I am grateful to Jean Bourgain, Elon Lindenstrauss and Pablo Shmerkin for their comments.

Research supported by NSF grant 0901534.

\section{References}

[1] Bedford, T., Fisher, A. M.: Ratio geometry, rigidity and the scenery process for hyperbolic Cantor sets. Ergodic Theory Dynam. Systems 17, 531-564 (1997) Zbl 0886.58089 MR 1452179

[2] Cooper, D., Pignataro, T.: On the shape of Cantor sets. J. Differential Geom. 28, 203-221 (1988) Zbl 0688.58025 MR 0961514

[3] Elekes, M., Keleti, T., Máthé, A.: Self-similar and self-affine sets: measure of the intersection of two copies. Ergodic Theory Dynam. Systems 30, 399-440 (2010) Zbl 1200.28005 MR 2599886

[4] Furstenberg, H.: Intersections of Cantor sets and transversality of semigroups. In: Problems in Analysis (Princeton, NJ, 1969), Princeton Univ. Press, Princeton, NJ, 41-59 (1970) Zbl 0208.32203 MR 0354562 
[5] Gavish, M.: Measures with uniform scaling scenery. Ergodic Theory Dynam. Systems 31, 33-48 (2011) Zbl 1225.37012 MR 2755920

[6] Hochman, M.: Dynamics on fractals and fractal distributions. Preprint (2009)

[7] Hochman, M., Shmerkin, P.: Local entropy and dimension of projections. Preprint

[8] Host, B.: Nombres normaux, entropie, translations. Israel J. Math. 91, 419-428 (1995) Zbl 0839.11030 MR 1348326

[9] Hunt, B. R., Kaloshin, V. Yu.: How projections affect the dimension spectrum of fractal measures. Nonlinearity 10, 1031-1046 (1997) Zbl 0903.28008 MR 1473372

[10] Johnson, A. S. A.: Measures on the circle invariant under multiplication by a nonlacunary subsemigroup of the integers. Israel J. Math. 77, 211-240 (1992) Zbl 0790.28012 MR 1194793

[11] Johnson, A. S. A., Rudolph, D. J.: Commuting endomorphisms of the circle. Ergodic Theory Dynam. Systems 12, 743-748 (1992) Zbl 0787.58027 MR 1200341

[12] Johnson, A., Rudolph, D. J.: Convergence under $\times_{q}$ of $\times_{p}$ invariant measures on the circle. Adv. Math. 115, 117-140 (1995) Zbl 0934.28010 MR 1351328

[13] Kalinin, B., Katok, A., Rodriguez Hertz, F.: New progress in nonuniform measure and cocycle rigidity. Electron. Res. Announc. Math. Sci. 15, 79-92 (2008) Zbl 1159.37316 MR 2457050

[14] Lindenstrauss, E.: Rigidity of multiparameter actions. Israel J. Math. 149, 199-226 (2005) Zbl 1155.37301 MR 2191215

[15] Maker, P. T.: The ergodic theorem for a sequence of functions. Duke Math. J. 6, 27-30 (1940) Zbl 0027.07705 MR 0002028

[16] Mattila, P.: Geometry of Sets and Measures in Euclidean Spaces. Cambridge Stud. Adv. Math. 44, Cambridge Univ. Press, Cambridge (1995) Zbl 0819.28004 MR 1333890

[17] Pesin, Y. B.: Dimension Theory in Dynamical Systems. Chicago Lectures in Math.. Univ. of Chicago Press, Chicago, IL (1997) Zbl 0895.58033 MR 1489237

[18] Rudolph, D. J.: $\times 2$ and $\times 3$ invariant measures and entropy. Ergodic Theory Dynam. Systems 10, 395-406 (1990) Zbl 0709.28013 MR 1062766

[19] Walters, P.: An Introduction to Ergodic Theory. Grad. Texts in Math. 79, Springer, New York (1982) Zbl 0475.28009 MR 0648108

[20] Xi, L.-F.: Lipschitz equivalence of self-conformal sets. J. London Math. Soc. (2) 70, 369-382 (2004) Zbl 1160.28305 MR 2078899 